\title{
FUNCTION THEORY AND MULTIPLICATIVE LINEAR FUNCTIONALS
}

\author{
BY \\ KENNETH HOFFMAN $\left({ }^{1}\right)$ AND HUGO ROSSI $\left({ }^{2}\right)$
}

1. Introduction. Let $m$ be a probability measure. In (complex) $L^{\infty}(m)$, we consider a subalgebra $A$, which contains the constant functions. We assume

(a) $m$ is multiplicative on $A$, that is,

$$
\int f g d m=\int f d m \int g d m, \quad f, g \in A ;
$$

(b) if $h$ is a non-negative function in $L^{1}(m)$ and

$$
\int f h d m=\int f d m, \quad f \in A,
$$

then $h=1$, almost everywhere.

If $1 \leqq p<\infty$, we let $H^{p}$ be the closure of $A$ in the Banach space $L^{p}(m)$. We define

$$
H^{\infty}=H^{2} \cap L^{\infty}(m) .
$$

The principal result of this paper is that $H^{\infty}$ is a logmodular algebra on the maximal ideal space of $L^{\infty}(m)$, i.e., that each real-valued function in $L^{\infty}(m)$ is the logarithm of the modulus of an invertible function in the algebra $H^{\infty}$.

This enables us to deduce from (a) and (b) the bulk of the generalized analytic function theory which is valid for logmodular algebras [8]. Srinivasan and Wang [10] have shown that this "function theory" follows if one assumes (a), (b), and

(c) $A+\bar{A}$ is dense in $L^{2}(m)$.

Lumer [9] has demonstrated the results under the assumption that $A$ is an algebra of continuous functions on a compact Hausdorff space, $m$ is a Borel measure on $X$ which is multiplicative on $A$, and no other positive measure on $X$ induces the same linear functional on $A$ as does $m$. In $\$ 5$, we shall comment on this function algebra setting.

2. Logmodularity. Let us abbreviate $L^{p}(m)$ to $L^{p}$. The elements of $L^{p}$ are equivalence classes of functions; however, we shall treat them as functions, and speak of the equality of two such functions-consistently

Received by the editors June 5, 1964 .

( $\left.{ }^{1}\right)$ Supported by NSF GP 2600.

(') Supported by NSF GP 170. 
omitting "almost everywhere with respect to $m$ ". We denote by $L_{R}^{p}$ the set of real-valued functions in $L^{p}$.

We retain the algebra $A$, which satisfies the conditions (a) and (b). In addition to the spaces $H^{p}$ and $H^{\infty}$, we define

$$
\begin{aligned}
& A_{0}=\left\{f \in A ; \int f d m=0\right\}, \\
& H_{0}^{p}=\left\{f \in H^{p} ; \int f d m=0\right\} .
\end{aligned}
$$

Note that (a) says that $A_{0}$ is an ideal in $A$, and (b) can be stated as follows:

(b') if $g \in L^{1}, g \geqq 0$ and $\int f g d m=0$ for all $f \in A_{0}$, then $g$ is constant. Since $m$ is multiplicative on $A$, we see directly that

$$
\int f g d m=\int f d m \int g d m
$$

for $f, g \in H^{2}$. In particular, $m$ is multiplicative on $H^{\infty}$. Also note that $H^{\infty}$ is a closed subalgebra of $L^{\infty}$ in the (essential) sup norm on $L^{\infty}$. Thus, if $h \in H^{\infty}$, then also $e^{h} \in H^{\infty}$ and $\int e^{h} d m=\exp [\mathcal{S} h d m]$.

Lemma 1. For $h$ a non-negative function in $L_{R}^{1}$, let $P$ be the orthogonal projection of 1 into the closure of $A_{0}$ in the Hilbert space $L^{2}(h d m)$. Then.

$$
|1-P|^{2} h=k \text {, }
$$

where $k$ is the constant

$$
k=\inf _{f \in A_{0}} \int|1-f|^{2} h d m=\inf _{f \in H_{0}^{\infty}} \int|1-f|^{2} h d m .
$$

Proof. First, define $k$ as the first infimum. Then, by definition of $P$,

$$
k=\int|1-P|^{2} h d m \text {. }
$$

Since $1-P$ is in the closure of $A$ in $L^{2}(h d m)$ then for any $f \in A_{0}, f(1-P)$ is in the closure of $A_{0}$. Thus, since $1-P$ is orthogonal to the closure of $A_{0}$,

$$
\int f(1-P)(1-\bar{P}) h d m=\int f|1-P|^{2} h d m=0
$$

for all $f \in A_{0}$. Since $|1-P|^{2} h \in L^{1}$, by $\left(\mathrm{b}^{\prime}\right),|1-P|^{2} h$ is constant, and that constant must be $k$. Finally we must show that the two infima are equal. Notice that $|1-P|^{2} h^{2}=k h \in L^{1}$, so $(1-P) h \in L^{2}$ and is orthogonal (now in $L^{2}$ ) to $A_{0}$. Thus $(1-P) h$ is orthogonal to $H_{0}^{\infty}$, so $1-P$ is orthogonal to $H_{0}^{\infty}$ in $L^{2}(h d m)$. Since $P$ is in the $L^{2}(h d m)$ closure of $A_{0}$, and thus of $H_{0}^{\infty}$, we see that $P$ is also the orthogonal projection of 1 into the $L^{2}(h d m)$ closure of $H_{0}^{\infty}$, so the two infima are equal. 
Lemma 2. For $u \in L_{R}^{\infty}$, there is a function $F$ in $H^{\infty}$ such that $\mathcal{S} F d m=1$ and $|F| e^{u}=k$ where $k>0$ and $k=\inf _{f \in A_{0}} \mathcal{S}|1-f|^{2} e^{u} d m$.

Proof. We apply Lemma 1 for $h=e^{u}$. In this case the $L^{2}(h d m)$ closure of $A_{0}$ is the same as $H_{0}^{2}$. Thus, since $1 \notin H_{0}^{2}, k>0$, and $P$ is in $H_{0}^{2}$. Thus $F=(1-P)^{2}$ is the desired function.

Lemma 3. Let $\phi$ be a linear functional on $L^{\infty}$ such that $\|\phi\|=1$ and $\phi(h)$ $=\int h d m$ for all $h \in H^{\infty}$. Then $\phi(g)=\int g d m$ for all $g \in L^{\infty}$.

Since $\|\phi\|=\phi(1)=1, \phi$ is a positive linear functional on $L^{\infty}$. Thus we can restate Lemma 3 as follows: The linear functional which $m$ defines on $H^{\infty}$ has a unique positive (i.e., norm-preserving) extension to a linear functional on $L^{\infty}$.

Proof. We shall prove that $\phi$ is absolutely continuous with respect to the linear functional which $m$ defines on $L^{\infty}$. Indeed, we assert that

$$
\phi(u) \leqq e \int u d m, \quad u \in L_{R}^{\infty}, u \geqq 0 .
$$

It suffices to prove this for characteristic functions; thus we may assume that $u$ is the characteristic function of an $m$-measureable set $E$. By Lemma 1 , there is an $F \in H^{\infty}$ such that $\int F d m=1$ and $|F| e^{u}=k$, where

$$
k=\inf _{f \in A_{0}} \int|1-f|^{2} e^{u} d m .
$$

Note that

$$
k \leqq \int e^{u} d m=1+(e-1) m(E) .
$$

Now let $h=k^{-1} F$. Then $h \in H^{\infty},|h|=e^{-u}$, and $\int h d m=k^{-1}$. Since $\phi$ agrees with $m$ on $H^{\infty}$,

$$
k^{-1}=\phi(h) \leqq \phi(|h|)=\phi\left(e^{-u}\right)=1+\left(e^{-1}-1\right) \phi(u),
$$

since $e^{-u}=1+\left(e^{-1}-1\right) u$. Thus

$$
\frac{e-1}{e} \phi(u) \leqq \frac{k-1}{k} \leqq \frac{(e-1) m(E)}{k} .
$$

But $k \geqq 1$, since

Thus

$$
k=|F| e^{u}=\int|F| e^{u} d m \geqq\left|\int F d m\right|=1 .
$$

$$
\frac{1}{e} \phi(u) \leqq m(E)
$$

as required. Thus $\phi$ is continuous in the $L^{1}$ norm, so there is a non-negative 
$p \in L^{\infty}$ such that $\phi(g)=\int g p d m$ for all $g \in L^{1}$. In particular, $\int g p d m$ $=\int g d m$ for all $g \in A$, so by assumption (b), $p=1$, and the lemma is proven.

THEOREM 4. $H^{\infty}$ is a logmodular algebra, on the maximal ideal space of $L^{\infty}$.

Proof. We must show that each $u \in L_{R}^{\infty}$ is the logarithm of an invertible element of $H^{\infty}$. Note that Lemma 2 almost does this, we need only show that the $F$ found there is invertible. To do this, we must further identify the constant $k$. For $u \in L_{R}^{\infty}$, define

$$
k(u)=\inf _{f \in A_{0}} \int|1-f|^{2} e^{u} d m .
$$

Clearly, if $u \leqq v$ then $k(u) \leqq k(v)$. Now if $u=\operatorname{Re} h$, with $h \in H^{\infty}$, then $k(u)=\exp [\mathcal{J} u d m]$. It suffices to show that $k(u)=1$ when $u=\operatorname{Re} h, h$ $\in H_{0}^{\infty}$. First, let $g=1-\exp (-(1 / 2) h)$. Then $g \in H_{0}^{\infty}$, and

$$
\int|1-g|^{2} e^{u} d m=\int\left|e^{-h / 2}\right|^{2} e^{\text {Reh }} d m=1,
$$

so $k(u) \leqq 1$. On the other hand, for any $f \in A_{0}$,

$$
\int|1-f|^{2} e^{u} d m \geqq\left|\int(1-f)^{2} e^{h} d m\right|=1 .
$$

Now let $u \in L_{R}^{\infty}$. By the above arguments, we have the following inequalities:

$$
\begin{aligned}
& \sup \left\{\int \operatorname{Re} h d m, h \in H^{\infty}, \operatorname{Re} h \leqq u\right\} \\
& \leqq \log k(u) \leqq \inf \left\{\int \operatorname{Re} h d m ; h \in H^{\infty}, \operatorname{Re} h \geqq u\right\} .
\end{aligned}
$$

By Lemma 3, the linear functional which $m$ defines on the real parts of $H^{\infty}$ functions has a unique extension to a positive linear functional on $L_{R}^{\infty}$. The necessary and sufficient condition for this uniqueness is that the left and right sides of (1) are both equal to $\int u d m$. Thus $\log k(u)=\int u d m$ for $u \in L_{R}^{\infty}$.

Now, by Lemma 2, there are $F, G \in H^{\infty}$ such that $\int F d m=\int G d m=1$ and $|F| e^{u}=k(u),|G| e^{-u}=k(-u)$. By the above $k(u) k(-u)=1$, so $|F G|=1$. But $\int F G d m=\int F d m \int G d m=1$. Thus $F G=1$. Therefore $h=k(u) F^{-1}=k(u) G$ is an invertible element of $H^{\infty}$ and $\log |h|=u$.

3. The $H^{p}$ theory. Given the situation described in the above section, it is possible to deduce, from the logmodularity of $H^{\circ}$, all the theorems concerning the spaces $H^{p}$ as in [8]. However, in doing this one loses sight of the given algebra $A$. Further, it has to be verified that if we replace our basic algebra $A$ by $H^{\infty}$, we get the same $H^{p}$ spaces, i.e. the closure of $H^{\infty}$ in $L^{p}$ is the same as that of $A$ in $L^{p}, 1 \leqq p<\infty$. Therefore we provide 
the following bridge between $A$ and the theory in [8].

Theorem 5 [Szegö]. If $h \in L_{R}^{1}, h \geqq 0$, then

$$
\inf _{f \in A_{0}} \int|1-f|^{2} h d m=\exp \left[\int \log h d m\right] .
$$

Proof. Because of Lemma 1, this follows from the corresponding theorem for $H^{\infty}[8]$. However, we can give a direct proof. First observe that, as in the proof of Theorem 4, we know the theorem for functions $h$ which are bounded above, and bounded away from zero. Now let $h \in L_{R}^{1}$ be such that $h \geqq m$ for some $m>0$. Let $h_{n}=\inf \{h, n\}$. Then $h_{n} \rightarrow h$ monotonically. Let $f \in A_{0}$. Then

$$
\int|1-f|^{2} h d m \geqq \int|1-f|^{2} h_{n} d m \geqq \exp \left[\int \log h_{n} d m\right] .
$$

Letting $n \rightarrow \infty$ we find

$$
\inf _{f \in A_{0}} \int|1-f|^{2} h d m \geqq \exp \left[\int \log h d m\right] .
$$

Now, let $P_{n} \in H_{0}^{\infty}$ be given by Lemma 1 so that

$$
\left|1-P_{n}\right|^{2} h_{n}=\exp \left[\int \log h_{n} d m\right] .
$$

Since $h_{n} \geqq m$ for all $n,\left|1-P_{n}\right|^{2} \leqq m^{-1} \exp [\mathcal{S} \log h d m]=M$ for all $n$. Now, for $\epsilon>0$, choose $n$ so that $\int\left(h-h_{n}\right) d m<M^{-1} \epsilon$. Then

$$
\begin{aligned}
\int\left|1-P_{n}\right|^{2} h d m & =\int\left|1-P_{n}\right|^{2} h_{n} d m+\int\left|1-P_{n}\right|^{2}\left(h-h_{n}\right) d m \\
& <\exp \left[\int \log h d m\right]+\epsilon .
\end{aligned}
$$

Thus

$$
\inf _{f \in H_{0}^{\infty}} \int|1-f|^{2} h d m \leqq \exp \left[\int \log h d m\right],
$$

so by Lemma 1 the theorem is proven for such an $h$. Now, for any $h \in L_{R}^{1}$, $h \geqq 0$, let $h_{m}=\sup \left\{h, m^{-1}\right\}$. Since $h_{m}$ decreases monotonically to $h$ as $m \rightarrow \infty$, so the derived result follows from the monotone convergence theorem.

Now to show that $H^{\infty}$ and $A$ have the same $H^{p}$ spaces, it suffices to show that $A$ is weak dense in $H^{\infty}$. Now, by Theorem 5.4 of [8], $H^{\infty}$ $+\bar{H}^{\infty}$ is dense in $L^{2}$, therefore, since $A$ is $L^{2}$ dense in $H^{\infty}$ by definition, $A+\bar{A}$ is dense in $L^{2}$. In particular, it follows: that if $f$ is in $L^{2}$, then $f$ is in $H^{2}$ if and only if $\int \mathrm{fgdm}=0$ for all $g \in A_{0}$. Now by repeating the proof of the corresponding theorem in [8], (Theorem 6.1) we prove that 
if $f$ is in $L^{1}$ and $\int f g d m=0$ for all $g \in A$, then $f=h_{1} h_{2}$ with $h_{1} \in H_{0}^{2}$, $h_{2} \in H^{2}$. But then, if $g \in H^{\infty}$, also $\int f g d m=0$. Thus $A$ is weak ${ }^{*}$ dense in $H^{\infty}$. Summarizing:

Theorem 6. $A$ is weak* dense in $H^{\infty}$, and hence the norm closure of $A$ in $L^{p}, 1 \leqq p<\infty$ is the same as that of $H^{\infty}$. Further, $A+\bar{A}$ is weak $k^{*}$ dense in $L^{\infty}$, and hence is norm dense in $L^{p}, 1 \leqq p<\infty$.

4. An example. An immediate consequence of our assumption (b) is that $A+\bar{A}$ is dense in $L^{1}$. After Lemma 2 is proven, this is the extent to which assumption (b) is needed. It may be thought perhaps more natural to assume only that $A+\bar{A}$ is dense in $L^{1}$; however this will not do. In fact it is not even enough for $A+\bar{A}$ to be dense in $L^{p}, 1 \leqq p \leqq 3$ as the following example shows.

For $R>r>0$, let $X=\{z \in C ;|z|=R\} \cup\{z \in C ;|z|=r\}=\Gamma_{R} \cup \Gamma_{r}$, and let $A$ be the algebra of continuous functions on $X$ which have analytic extensions to the annulus $R=\{z \in C ; r<|z|<R\}$. Let $d \theta$ be the measure given by $d z / z$ on $X$, and let $E$ be the function

$$
E(x)= \begin{cases}1 & \text { if } x \in \Gamma_{R}, \\ -1 & \text { if } x \in \Gamma_{r} .\end{cases}
$$

It is well-known that if $g \in L_{R}^{1}$ and $\int f g d \theta=0$ for all $g \in A$, then $f=k E$, where $k$ is a constant.

Now let $p$ be such that $r<|p|<R$, and let $P$ be the Poisson kernel for $p$, i.e.,

$$
g(p)=\int_{X} g P d \theta
$$

for all $g \in A+\bar{A}$. $P$ is a strictly positive, real-analytic function on $X$. Let $\lambda=\inf \{P(z) ;|z|=R\}$. The measure $d m=(P-\lambda E) d \theta$ is clearly a probability measure which is multiplicative on $A \cdot P(P-\lambda E)^{-1}$ is a positive $L^{1}(d m)$ function which annihilates $A_{0}=\{f \in A ; f(p)=0\}$, so assumption $\left(b^{\prime}\right)$ does not hold (in fact it is clear that none of the desired theorems holds).

Suppose that $g \in L_{R}^{p}(d m)$ and $\int f g d m=0$ for all $f \in A$. Then

$$
\int f g(P-\lambda E) d \theta=0
$$

for all $f \in A$, so $g(P-\lambda E)=k E$ for some constant $k$. If $g$ is not identically zero, then $k \neq 0$, so $g \in L_{R}^{p}(d m)$ implies $|g|^{p}(P-\lambda E)=k^{p}(P-\lambda E)^{1-p}$ $\in L_{R}^{1}(d \theta)$. Now the integrability of $(P-\lambda E)^{1-p}$ depends only on its behavior on the curve $\Gamma_{R}$ at the point where $P$ attains its minimum. Since $P$ is differentiable there, the integrability of $(P-\lambda E)^{1-P}$ implies that of $x^{2(1-p)}$ in $L^{1}(d x)$ in a neighborhood of the origin in $R$. Thus $2(1-p)<1$, or $p<3 / 2$. Thus, if $p \geqq 3 / 2$, there is no nonzero $g \in L_{R}^{p}(d m)$ such that 
$\int f g d m=0$ for all $f \in A$. Thus, for $q \leqq 3, A+\bar{A}$ is dense in $L^{q}(d m)$.

5. The case that $A$ is a closed subalgebra of $C(X)$. Now suppose that $X$ is a compact Hausdorff space, $m$ is a Borel probability measure on $X$, and $A$ is a closed subalgebra of $C(X)$ containing the constants, on which $m$ is multiplicative. Thus $m$ is a representing measure for some complex homomorphism $\phi$ of the algebra $A$. We assume also (b), which says that any representing measure for $\phi$ which is absolutely continuous with respect to $m$ is equal to $m$. As a consequence, we have $A+\bar{A}$ dense in $L^{2}(m)$. Now, as in [11], we can prove that the set of complex homomorphisms of $A$ which are bounded on $H^{2}$ consists of either $\phi$ alone, or is an analytic disc. Precisely, we obtain the following result.

THEOREM 7. Let $A$ be a sup norm algebra on the compact Hausdorff space $X$, and let $\phi$ be a complex homomorphism of $A$. Suppose $m$ is a representing measure (on $X)$ for $\phi$ which has the property that no other representing measure for $\phi$ is absolutely continuous with respect to $m$. Let $D$ be the set of complex homomorphism $\psi$ of $A$ which are bounded on $H^{2}(m)$, i.e.,

$$
|\psi(f)|^{2} \leqq K \int|f|^{2} d m, \quad f \in A,
$$

for some constant $K$. Then, if $D \neq\{\phi\}$, there exists a one-one map $\tau$ from the open unit disc onto $D$ such that $f \circ \tau$ is analytic for every $f \in A$.

Proof. [8, Theorem 7.4].

This last theorem is part of a general attempt to place analytic structures on subsets of the maximal ideal space of a sup norm algebra. From this point of view, the result has some defects. First, if $D=\{\phi\}$, we have not produced any nontrivial analytic structure. This may happen even if there exists some nontrivial analytic structure passing through $\phi$; for example, by making a poor choice of representing measure $m$. However, if $m$ is the unique measure representing $\phi$, these difficulties disappear.

In [6], Gleason introduced equivalence classes of homomorphisms which he called "parts". Two homomorphisms $\phi$ and $\psi$ are in the same part if

$$
|\phi(f)-\psi(f)| \leqq c\|f\|, \quad f \in A,
$$

where $c$ is a constant, $0<c<2$. The parts are the largest possible analytic subsets of the maximal ideal space, in the sense that any subset of that space which can be endowed with the structure of an analytic space lies wholly in one part. Thus we can ask for conditions making the following statement true:

${ }^{*}$ ) If $\phi$ is a complex homomorphism of $A$, then the set $D$ of the previous theorem is precisely the Gleason part which contains $\phi$.

Gleason showed that $\left(^{*}\right)$ is true for Dirichlet algebras. Lumer [9] has extended this to any algebra for which each complex homomorphism of $A$ 
has a unique representing measure. Now, a recent result of Bishop [3] says that if $\phi$ and $\psi$ are two complex homomorphisms of a sup norm algebra $A$ which are in the same part, then given a measure $\nu$ representing $\psi$, there is a measure $\mu$ representing $\phi$ such that $\nu$ is absolutely continuous with respect to $\mu$ and $d \nu / d_{\mu}$ is bounded. Using this we obtain the following result.

TheOREM 8. Let $A$ be a sup norm algebra on the compact Hausdorff space $X$, and suppose $\phi$ is a complex homomorphism which has a unique representing measure. Then, if the Gleason part $P$ of $\phi$ is larger than $\{\phi\}$, there exists a one-one map $\tau$ from the open unit disc onto $P$ such that $f \circ \tau$ is analytic for every $f \in A$. In addition, every $\psi \in P$ has a unique representing measure.

Proof. Let $m$ be the measure representing $\phi$. Let $\psi \in P$, and let $\mu$ be a measure representing $\psi$. By Bishop's result, $d_{\mu}=p d m$ and $p \in L^{\infty}(m)$. Thus, for $f \in A$

$$
|\psi(f)|=\left|\int f p d m\right|^{2} \leqq\|p\| \int|f|^{2} d m,
$$

so $\psi \in D$. Thus $D=P$, so the first statement follows from Theorem 7 . Suppose $\mu^{\prime}$ is another measure representing $\psi$. Then $d \mu^{\prime}=p^{\prime} d m$, and $p^{\prime}$ $\in L^{\infty}(m)$. Thus $\left(p-p^{\prime}\right) d m=\mu-\mu^{\prime}$, so annihilates $A$. Since $p, p^{\prime}$ are bounded, for $\epsilon>0$ small enough, $\left[1+\epsilon\left(p-p^{\prime}\right)\right] d m$ is a positive measure and represents $\phi$. Thus $1+\epsilon\left(p-p^{\prime}\right)=1$, so $p=p^{\prime}$ and $\mu=\mu^{\prime}$.

\section{Bibliography}

1. R. Arens and I. M. Singer, Generalized analytic functions, Trans. Amer. Math. Soc. 81 (1956), 379-395.

2. A. Beurling, On two problems concerning linear transformations in Hilbert space, Acta Math. 81 (1949), 239-255.

3. E. Bishop, Representing measures for points in a uniform algebra, Bull. Amer. Math. Soc. 70 (1964), 121-122.

4. S. Bochner, Boundary values of analytic functions in several variables and of almost periodic functions, Ann. of Math. 45 (1944), 686-707.

5. Generalized conjugate and analytic functions without expansions, Proc. Nat. Acad. Sci. U.S.A. 45 (1959), 855-857.

6. A. Gleason, Function algebras, Seminars on Analytic Functions, Institute for Advanced Study, Princeton, N. J., 1957.

7. H. Helson and D. Lowdenslager, Prediction theory and Fourier series in several variables, Acta Math. 99 (1958), 165-202.

8. K. Hoffman, Analytic functions and logmodular Banach algebras, Acta Math. 108 (1962), 271-317.

9. G. Lumer, Analytic functions and Dirichlet problem, Bull. Amer. Math. Soc. 70 (1964), 98-104.

10. T. P. Srinivasan and J.-K. Wang, Weak* Dirichlet algebras (to appear).

11. J. Wermer, Dirichlet algebras, Duke Math. J. 27 (1960), 373-382.

Massachusetts Institute of TechNology,

Cambridge, Massachusetts

BRANDEIS UNIVERSITY,

Waltham, Massachusetts 